\title{
Modified Stoppa approach for acetabular fracture fixation - reduction techniques
}

\author{
Dhurvas Ramlal Ramprasath $^{1 *}$, S. Vetrivel Chezain ${ }^{2}$, Iniya Prasanna Raajendiren ${ }^{3}$ \\ ${ }^{\mathbf{1}}$ Associate Professor, ${ }^{2}$ Director and Professor, ${ }^{\mathbf{3}}$ Junior Resident, Institute of Orthopaedics and Traumatology, Coimbatore Medical College \\ and Hospital, Coimbatore, Tamil Nadu, India \\ *Corresponding Author: Dhurvas Ramlal Ramprasath \\ Email: dhurvasramprasath@gmail.com
}

\begin{abstract}
Introduction: Since acetabular fractures are intra-articular, accurate anatomic reduction is essential to achieve a good functional outcome. Among the various surgical approaches, ilioinguinal and modified Stoppa approaches are the only available intra-pelvic approaches. Even though ilioinguinal approach is more commonly used, modified Stoppa approach is becoming more popular due to the possibility of visualising and reducing quadrilateral plate fragments as well as fixation of posterior column through anterior approach. The aim of our study was to assess the efficacy of various reduction tools/ techniques for reduction and fixation of fractures operated using modified Stoppa approach.

Materials and Methods: We have operated on 16 patients with acetabular fractures through modified Stoppa approach in the age group of 19 years to 70 years (mean 42 years) with male female ratio of 13:3. In patients requiring combined approach, the anterior approach was done first. In all the patients, modified Stoppa was combined with a lateral window. We have used various reduction techniques including transtrochanteric Schanz pin, intra-focal elevators, ball spike, bone hook, iliac crest Schanz pin, Farabeuf forceps, undercontoured plate, etc. Results: Matta's radiographic reduction criteria showed anatomic reduction in 10 cases. Clinical assessment using Merle d' Aubigne scoring showed excellent results in 9 cases and good results in 6 cases. Follow up was done for a maximum period of six months. Conclusion: Modified Stoppa approach provides better visualisation of quadrilateral surface and sciatic buttress when compared to the ilioinguinal approach. The use of appropriate reduction techniques helps in achieving good reduction of anterior column, at the same time provides opportunity to fix the posterior column from the anterior aspect using anterior to posterior column screws, obviating the need for a separate posterior approach.
\end{abstract}

Keywords: Modified Stoppa, Acetabulum, Anterior column, Reduction, Fixation, Quadrilateral plate.

\section{Introduction}

Since acetabular fractures are intraarticular and involve major weight bearing joint, accurate anatomic reduction is essential to achieve a good functional outcome. The surgical approaches include Kocher Langenbeck, Iliofemoral, Ilioinguinal, combined anterior and posterior approaches, extended iliofemoral, transtrochanteric and triradiate approaches. ${ }^{1-9}$ Of these approaches, only the ilioinguinal is intrapelvic. Since its original description by Letournel in 1961, the ilioinguinal approach has gained popularity for fixation of anterior wall and column fractures, anterior fractures associated with a posterior hemitransverse component, and even both column fractures.

The modified Stoppa approach is an intrapelvic approach initially used for inguinal hernia surgery by Rives et al and Stoppa et al (in 1984). ${ }^{10,11}$ Hirvensalo et al (in 1993), ${ }^{12}$ and later Cole and Bolhofner (in 1994) ${ }^{13}$ used the same approach with some modifications to visualise the quadrilateral plate and the sciatic buttress for fixation of acetabular fractures, and duly credited Stoppa by describing the technique as modified Stoppa approach. Ilioinguinal and modified Stoppa approaches are the currently available intrapelvic approaches, of which ilioinguinal is more commonly used and its outcomes have been reported more extensively. ${ }^{14}$ Sagi et al $^{15}$ additionally described the use of Smith Peterson approach (and/or an anterior superior iliac spine osteotomy) to better visualise the anterior portion of the joint and anterior wall. Qureshi et $\mathrm{al}^{16}$ further described steps to "infrapectineal plating" as a technical trick. In the evolution of modified Stoppa approach, many contributors addressed anatomy and safety concerns, specific technical issues, clinical outcomes, and prognosis mainly through retrospective cohort studies. The aim of our study was to assess the efficacy of various reduction tools/ techniques for reduction and fixation of fractures operated using modified Stoppa approach.

\section{Materials and Methods}

This is a retrospective study done in a tertiary care hospital from January 2018 to February 2019 involving 16 patients who had undergone modified Stoppa approach for acetabular fractures. There were 13 males and 3 females in the age group of 19 years to 70 years (mean 42 years). There were a total of 67 acetabular fractures, of which modified Stoppa approach was indicated in 18 patients. Letournel and Judet classification system was used to classify the fractures. After excluding two patients who were not fit for surgery (managed with skeletal traction alone), we had 16 patients operated through modified Stoppa approach. Informed written consent was obtained from all the patients. There were 11 cases of anterior column with posterior hemitransverse, three patients having anterior column and two cases of transverse fractures. We have used combined anterior and posterior approach in stages in 11 patients. In all the cases requiring combined approach, the anterior approach was done first. In all the 16 patients, modified Stoppa was combined with a lateral window (iliofemoral approach). 


\section{Procedure}

Through a curvilinear Pfannenstiel incision $2 \mathrm{cms}$ proximal to the pubic symphysis, the rectus sheath was incised horizontally and the linea alba was split to expose the retropubic space of Retzius. Using finger dissection, bladder was freed from the pubis; corona mortis was identified over the pubic ramus and ligated; obturator nerve and vessels were visualised (Fig. 1). The periosteum overlying pubic ramus was incised along with iliopectineal fascia to free the external iliac vessels and iliacus muscle anteriorly and obturator neurovascular bundle inferiorly. Deavor's retractor was used to retract the peritoneum proximally so that sciatic buttress was exposed. Now the fracture was visualised and reduced by various techniques (both direct and indirect methods) mentioned below. C arm was used to aid in anatomic reduction.

\section{Reduction Techniques}

1. Pre-operative upper tibial pin traction provided pain relief, at the same time realigning the fracture fragments.

2. A $5.5 \mathrm{~mm}$ Schanz pin introduced through the trochanter into the head of femur was used to apply lateral traction with the help of a $T$ handle. In cases of protrusio, the head of femur was retrieved from the pelvis with a schuck sound (Fig. 2). In other cases, the anterior/ posterior column fragments which were pushed by the head of femur got reduced after the lateral traction was applied.

3. A Hohman retractor/ Cobb's elevator/ $\mathrm{K}$ wire was inserted intrafocally into the fracture and manipulated in a fashion so as to reduce the step created by the overlapping fragments (Fig. 3).

4. A ball spike (straight/ curved) was used to push the fragments which were lifted off from the fracture site. In cases of quadrilateral plate fracture displacement, ball spike was used to push the quadrilateral plate into position (Fig. 4).

5. A bone hook introduced through the lateral iliofemoral window was used to pull the larger displaced pelvic brim fragment (Fig. 5).

6. Manual longitudinal traction applied to the lower limb was also used to reduce the fracture fragments.

7. A $5.5 \mathrm{~mm}$ Schanz pin inserted into the iliac crest was used to manipulate the posterior column fragments (Fig. 6).

8. Manual pelvic compression was sometimes used to reduce the iliac wing fragments.

9. A Farabeuf forceps was used as a push-pull technique to reduce and approximate the iliac wing fracture fragments, by applying one screw on either side of the fracture.

10. In cases where there was plastic deformation of the pectineal eminence/ quadrilateral plate, an undercontoured reconstruction plate was applied on the bone, so that while tightening the screws applied on either side of the fracture, the plate pushed the deformed fragments into original anatomic position (Fig. 7).

11. An oblique Matta's clamp was used in some cases to reduce, compress and hold the fragments in position to facilitate plate fixation.

12. A pelvic bone model was kept adjacent to the operating table to help the surgeon in gaining better orientation of the bony contours. Moreover bone model was used to contour the plates preoperatively (Fig. 8).

13. Catheterisation of bladder, pre-operative enema, flexion of the hip and knee to $30^{\circ}$ (by placing a bolster under the ipsilateral knee) - all manoeuvres helped for better visualisation and relaxation of intra-pelvic structures, thus aiding in fracture reduction and stabilisation, and also avoiding injuries to the neurovascular structures.

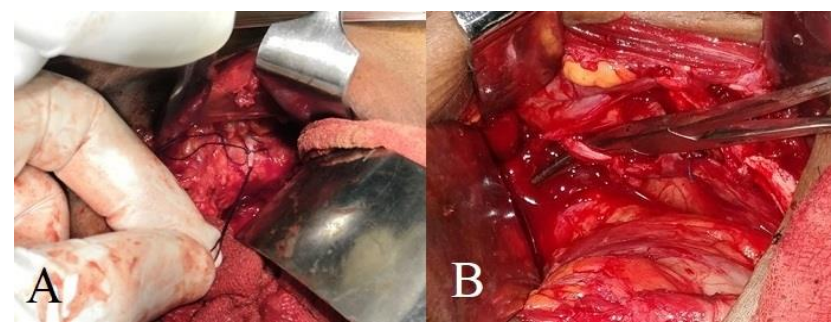

Fig. 1: Surgical exposure showing A): Corona mortis identified and ligated B): Obturator nerve isolated and protected

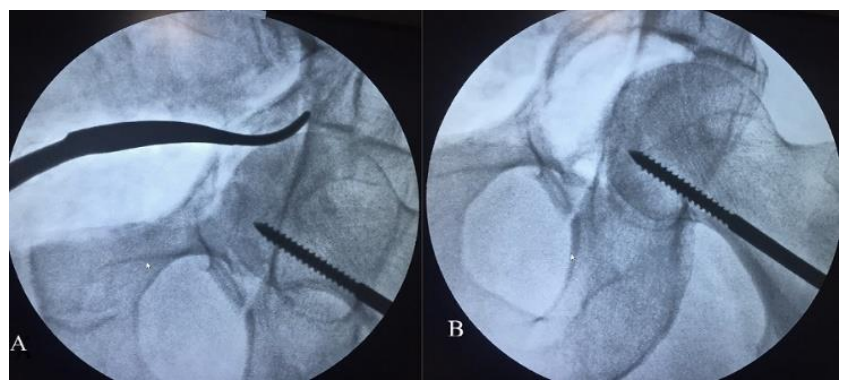

Fig. 2: Transtrochanteric Schanz pin inserted into head of femur; A) Before lateral traction; B) After lateral traction

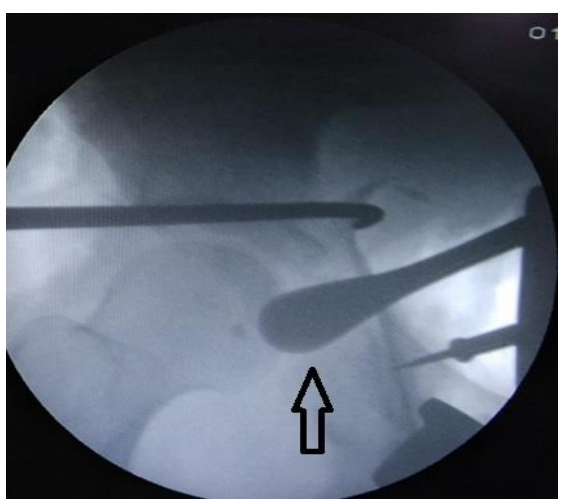

Fig. 3: Intrafocal instrumentation (Cobb's elevator) to achieve fracture reduction 


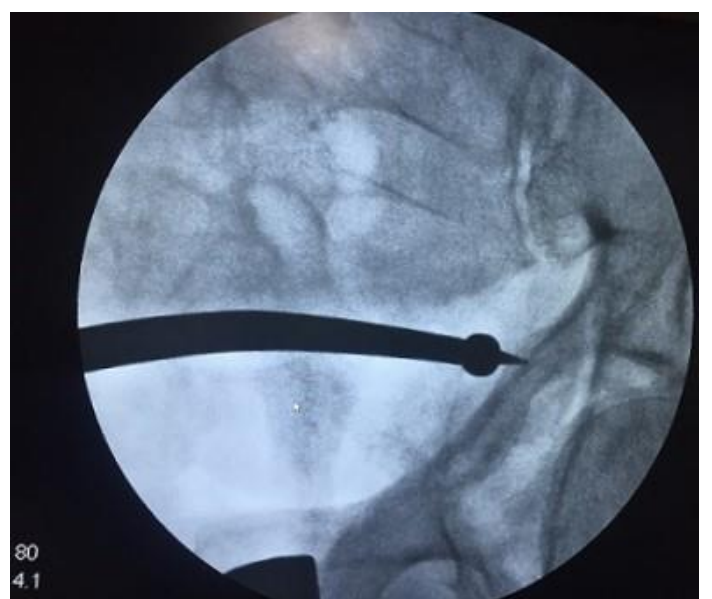

Fig. 4: A picador ball spike pushing the displaced quadrilateral plate

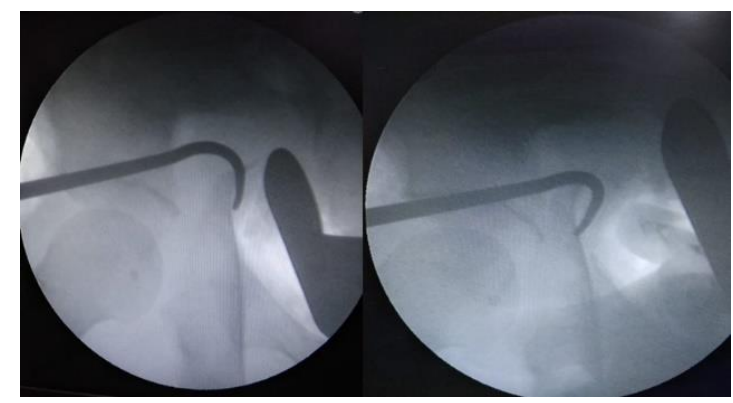

Fig. 5: Bone hook to pull the pelvic brim

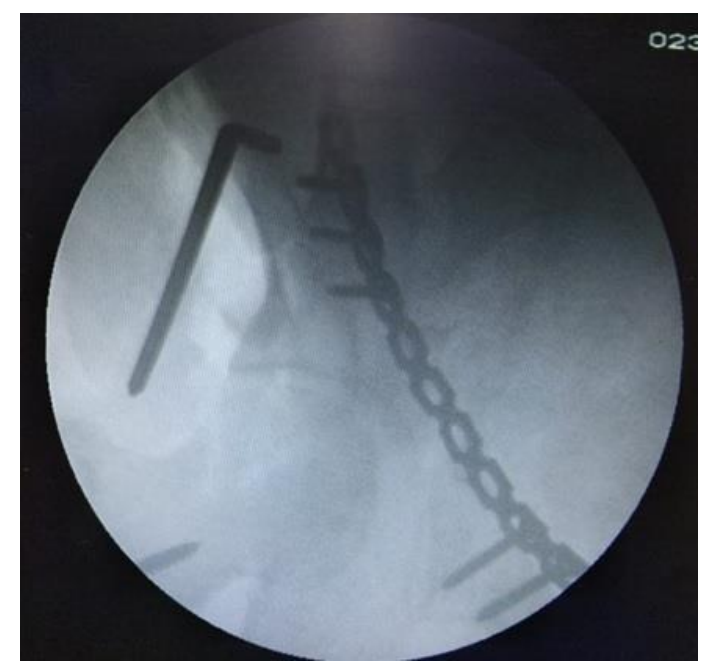

Fig. 6: Schanz pin inserted into iliac crest (in this case, bent due to manipulation)

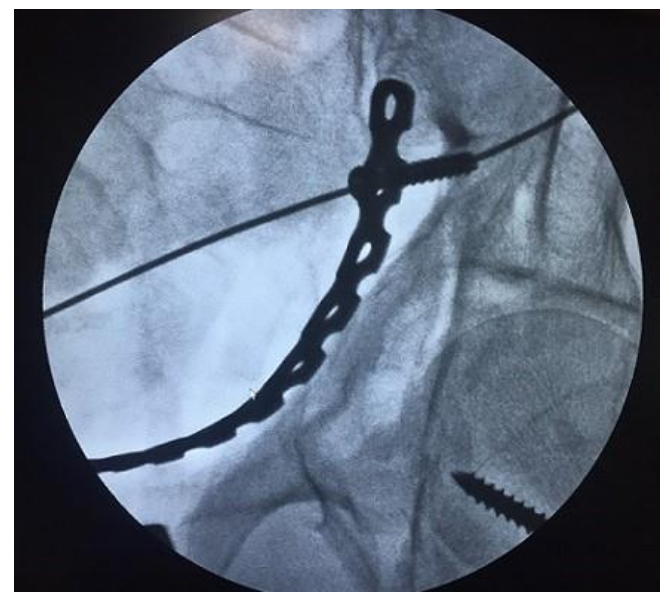

Fig. 7: Undercontoured reconstruction plate has been used to push the quadrilateral plate. Screw is inserted into the sciatic buttress

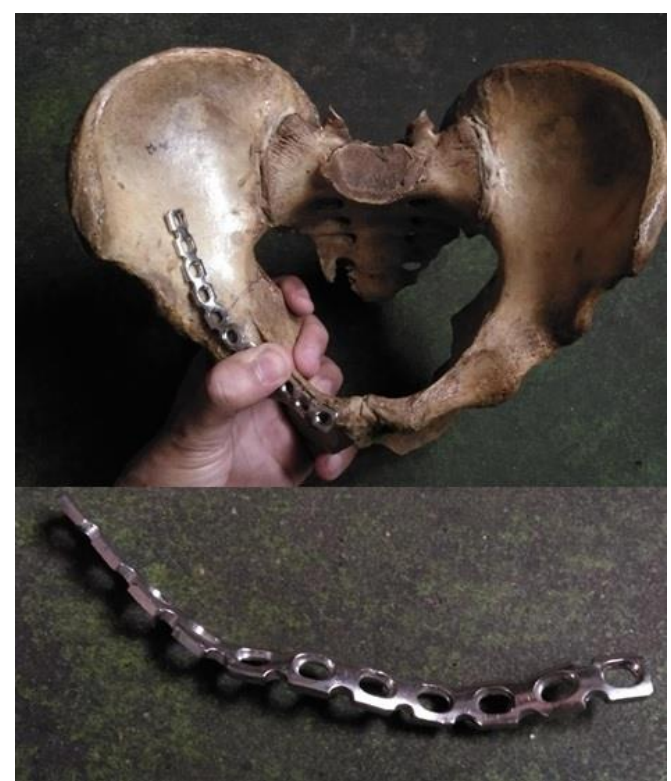

Fig. 8: Contouring the plate preoperatively using a cadaveric bone model

\section{Post Op protocol}

Oral feed was started once bowel sounds were heard and patient passed flatus. DVT prophylaxis was initiated using pharmacologic agents six hours after surgery. CT scan was taken to confirm the reduction as well as to rule out intraarticular screw placement. Follow up x-rays were taken two weeks, four weeks, six weeks and 12 weeks post operatively. Radiological assessment was done using Matta's criteria, and clinical assessment was done using Merle d' Aubigne scoring. Non-weight bearing mobilisation was started on second post-operative day and continued till 12 weeks.

\section{Results}

There were 16 patients with male female ratio of 13:3 in the age group of 19 years to 70 years (mean 42 years). The time 
from injury to surgery ranged from 6 to 14 days (mean 9 days). The success of reduction techniques was assessed using intra-operative $\mathrm{C}$ arm images as well as post-operative x-rays. Matta's radiographic reduction criteria showed anatomical reduction in 10 cases, imperfect results in 4 cases and poor results in 2 cases. Clinical assessment using Merle d' Aubigne scoring showed excellent results in 9 cases, good results in 6 and fair results in 1 case. Follow up was done for a maximum of six months (four to six months).

\section{Discussion}

Referred to by various names, the anterior intrapelvic approach (also known as AIP, Stoppa, modified Stoppa, extended Pfannenstiel, ilioanterior) aims to expose the medial intrapelvic surface (including quadrilateral plate and sciatic buttress) of the innominate bone for repair of acetabular and pelvic ring injuries. ${ }^{17}$ This approach utilises preperitoneal/ retroperitoneal interval to expose the inner aspect of pelvis. This approach provides access to pubic body, superior ramus, pubic root, the ilium above and below the pectineal line, the quadrilateral plate, the medial aspect of the posterior column, the sciatic buttress and the anterior sacroiliac joint. ${ }^{15}$ For access to the upper ilium and crest, a second incision following the upper limb of the approach described by Smith Peterson is used. An osteotomy of the anterior superior iliac spine improves the access to anterior wall - pubic root region. Sagi et $\mathrm{al}^{15}$ reported that $60 \%$ of their patients required exposure of the lateral window. In our study, we have performed a lateral window in all of our cases for placement of instruments for reduction and for fixation purpose.

Several reduction techniques have been described in literature. ${ }^{13,17,18}$ Reduction manoeuvres, however, often remain the most time consuming and difficult part of the procedure. ${ }^{13,19}$ Of these, lateral retraction of the femoral head with a trochanteric traction pin is an important technique, which facilitates reduction by eliminating deforming forces on the medially displaced fracture. This manoeuvre also releases tension on the obturator neurovascular bundle during retraction. ${ }^{18} \mathrm{~A}$ pelvic collinear reduction clamp, standard Matta pelvic reduction clamp can be used to reduce the column fractures. A picador ball spike pusher is a very useful instrument to reduce the anterior fracture. We have used ball spike in most of our cases. The use of a bone hook in the sciatic notch to bring forward the posterior column has been reported in literature. ${ }^{17} \mathrm{We}$ have used a bone hook introduced through the lateral window to reduce anterior column fracture in two of our cases. An undercontoured plate has been used by Qureshi et al ${ }^{16,18}$ to reduce and fix fractures involving quadrilateral surface. We have also used an undercontoured reconstruction plate buttressing the quadrilateral surface with screws inserted into the posterior column and posterior surface of pubic ramus. In addition to the techniques described above in the literature, we have used other techniques like 1) Schanz screw in the iliac crest to manipulate the posterior column, 2) Intrafocally placed Hohman/ Cobbs elevator/ K wire to lever out the impacted fragments, 3) Farabeuf forceps to apply compression on two screws, one each on the either side of fracture 4) Manual longitudinal traction of the lower limb.

The use of various reduction techniques helps in achieving anatomic reduction quickly, thus decreasing the duration of surgery as well as blood loss. Moreover, proper placement of instruments helps in avoiding injury to the neurovascular structures and also prevents intraarticular placememt of screw. Accoding to Cole and Bolhofner ${ }^{13}$, in fractures requiring both an anterior and posterior approach, the anterior was always performed first to allow reduction of significant posterior column, transverse, and medial wall fractures from the inside, essentially restoring anatomicity of the acetabulum prior to performing the posterior approach. This leads to a minimisation of dissection when a subsequent posterior approach is done.

The reduction quality translates itself to the radiograph results as well as clinical results. According to Ha Yong Kim et $a l,{ }^{14}$ out of 22 patients treated by a Stoppa approach, anatomic reduction was achieved in 17 cases, good and excellent radiographic results were obtained in 18 cases, and good and excellent clinical results were obtained in 16 cases. The radiographic results were slightly better than the clinical results. Marsh et al ${ }^{14,20}$ stated that the most important factor that determines the clinical results was damage to the joint cartilage, which would induce degenerative changes in the joints even if anatomic reduction was achieved. Since damage to articular cartilage is a non-modifiable factor, we recommend that modifiable factors like anatomic reduction should be given priority to achieve maximum possible functional results. Various reduction techniques mentioned above will help in achieving this goal.

Complications due to intraarticular screw placement can be avoided by following the "safe zone" mapping of Guy et $\mathrm{al}^{21}$ and the "safe and dangerous zone" mapping of Zang et al. ${ }^{22}$ Complications like neurovascular injury can be avoided by using appropriate instruments and safe retraction of the pelvic structures. In our study we have not encountered any major complications. Complications due to malreduction are common if the fixation is done after three weeks of injury. Cole and Bolhofner therefore advise that fixation be done before three weeks to obtain good results with respect to reduction and function.

\section{Conclusion}

In acetabular fractures requiring an anterior approach, modified Stoppa approach provides better visualisation of quadrilateral surface and sciatic buttress when compared to the ilioinguinal approach. A lateral window markedly improves the quality of reduction and fixation. The use of appropriate reduction techniques helps in achieving good reduction of anterior column, at the same time provides 
opportunity to fix the posterior column from the anterior aspect using anterior to posterior column screws, obviating the need for a separate posterior approach. This study shows that the results of fixation through modified Stoppa approach can be improved by using various reduction techniques.

\section{Limitation of our Study}

We have a relatively small sample size and a short follow up. The functional results like post-traumatic arthritis could not be analysed in our study. Application of appropriate reduction techniques and adequate follow up by further studies may provide the comparison between good radiographic results and corresponding clinical results.

\section{Conflict of interest: Nil}

\section{Acknowledgements: Nil}

\section{References}

1. Letournel E. Acetabulum fractures: Classification and management. Clin Orthop 1980;151:81.

2. Bray TJ, Esser M, Fulkenson L: Osteotomy of the trochanter in open reduction and internal fixation of acetabular fractures. $J$ Bone Joint Surg 1987;69A:711.

3. Goulet JA, Bray TJ. Complex acetabular fractures. Clin Orthop 1989;9:24

4. Kebaish AS, Roy A, Rennie W: Displaced acetabular fractures: Long-term follow-up. J Trauma 1991;31:1539.

5. Letournel E. Les fractures du cotyle, etude d'une serie de 75 cas. J Chir 1961;82:47.

6. Mears DC, Rubash HE: Extensile exposure of the pelvis. Contemp Orthop 1983;6:21.

7. Reinert CM, Bosse MJ, Poka A. A modified extensile exposure for the treatment of complex or malunited acetabular fractures. J Bone Joint Surg 1988;70A:329.

8. Routt MLC, Swiontkowski MF. Operative treatment of complex acetabular fractures. Combined anterior and posterior exposures during the same procedure. J Bone Joint Surg 1990;72A:897.

9. Winquist RA. Open reduction and internal fixation of central acetabular fractures by anterior and posterior approach. Orthop Trans 1982;6:93.

10. Stoppa RE, Rives JL, Warloumont CR, Palot JP, Verhaeghe PJ, Delattre JF. The use of Dacron in the repair of hernias of the groin. Surg Clin North Am 1984;64(2):269-85.

11. Rives J, Stoppa R, Fortesa L, Nicaise H. Dacron patches and their place in surgery of groin hernia: 65 cases collected from a complete series of 274 hernia operations. Ann Chir 1968;22(3):159-71.

12. Hirvensalo E, Lindahl J, Bostman O. A new approach to the internal fixation of unstable pelvic fractures. Clin Ortho Relat Res 1993;297:28-32.

13. Cole JD, Bolhofner BR. Acetabular fracture fixation via a modified Stoppa limited intrapelvic approach. Description of operative technique and preliminary treatment results. Clin Ortho Relat Res 1994;305:112-23.

14. Ha Yong Kim, Dae Suk Yang, Chang Kyu Park, Won SIk Choy. Modified Stoppa approach for surgical treatment of acetabular fracture. Clin Orthop Surg 2015;7:29-38.

15. Sagi HC, Afsari A, Dziadosz D. The anterior intra-pelvic (modified Rives-Stoppa) approach for fixation of acetabular fractures. J Orthop Trauma 2010;24:263-70.

16. Qureshi AA, Archdeacon MT, Jenkins MA. Infrapectineal plating for acetabular fractures: a technical adjunct to internal fixation. J Orthop Trauma 2004;18:175-8.

17. Pierre Guy. Evolution of the anterior intrapelvic (Stoppa) approach for acetabular fracture surgery. J Orthop Trauma 2015;29:S1-5.

18. Michael T. Archdeacon, MD, Namdar Kazemi, MD, Pierre Guy, MD, Henry C.Sagi, MD. The modified Stoppa approach for acetabular fracture. J Am Acad Orthop Surg 2011;19:170175.

19. Tile M, Helfet DL, Kellam JF, Letournel E, and Matta JM: Symposium: Management of acetabular fractures- Part II. Contemp Orthop 1992;25:389.

20. Marsh JL, Buckwalter J, Gelberman R. Articular fractures: does an anatomic reduction really change the result? J Bone Joint Surg Am 2002;84(7):1259-71.

21. Gay P, Al Otaibi M, Harvey EJ. The "safe zone" for extraarticular screw placement during intra-pelvic acetabular surgery. J Orthop Trauma 2010;24:279-83.

22. Zhang S, Su W, Luo Q. Measurement of the "safe zone" and the "dangerous zone" for the screw placement on the quadrilateral surface in the treatment of pelvic and acetabular fractures with Stoppa approach by computational 3D technology. Biomed Res Int 2014. Available at: http://dx.doi.org/10.1155/2014/386950. Accessed May 14,2014

How to cite this article: Ramprasath DR, Chezain SV, Raajendiren IP. Modified Stoppa approach for acetabular fracture fixation - reduction techniques. Indian J Orthop Surg 2019;5(2):106-10. 